\title{
Precise predictions for Higgs-boson production in association with top quarks
}

\author{
Ansgar Denner* \\ Universität Würzburg, Institut für Theoretische Physik und Astrophysik, D-97074 Würzburg, \\ Germany \\ E-mail: denner@physik.uni-wuerzburg.de
}

\section{Robert Feger}

Universität Würzburg, Institut für Theoretische Physik und Astrophysik, D-97074 Würzburg, Germany

E-mail: robert.feger@gmail.com

\section{Andreas Scharf}

Universität Würzburg, Institut für Theoretische Physik und Astrophysik, D-97074 Würzburg, Germany

E-mail: ascharfephysik.uni-wuerzburg.de

\begin{abstract}
We analyse the production of a Higgs boson in association with a top-antitop-quark pair in the Standard Model at the LHC. Considering the final state consisting of four $b$ jets, two jets, one identified charged lepton and missing energy, we examine the irreducible background for the production rate and several kinematical distributions. While $\mathrm{ttH}$ production and decay is roughly a fourth of the full process for the final state specified above, ttbb production constitutes the main contribution with about $92 \%$. Surprisingly, interference effects result in a reduction of the crosssection by five per cent. Furthermore, we consider NLO QCD corrections for the production of a Higgs boson, two charged leptons, two neutrinos, and two b jets. We discuss the size of the corrections and the scale dependence for the integrated cross section and different distributions. For the integrated cross section we find a $K$ factor of 1.17 and a reduction of the scale dependence from $30 \%$ at leading order to $5 \%$ at next-to-leading order.
\end{abstract}

12th International Symposium on Radiative Corrections (Radcor 2015) and LoopFest XIV (Radiative Corrections for the LHC and Future Colliders)

15-19 June, 2015

UCLA Department of Physics \& Astronomy Los Angeles, USA

\footnotetext{
*Speaker.
} 


\section{Introduction}

After the discovery of a Higgs boson with a mass around $125 \mathrm{GeV}$ by the CMS and ATLAS collaborations $[1,2]$, its properties need to be precisely investigated using all accessible Higgs production and decay modes. The production of a Higgs boson in association with a top-quark pair is of particular interest as it allows to directly access the top-quark Yukawa coupling. In the ongoing run 2 of the LHC the determination of the $t \bar{t} \mathrm{H}$ signal and the potential measurement of the top-quark Yukawa coupling will be pursued and corresponding theoretical predictions are required.

Next-to-leading-order (NLO) QCD corrections for the production of a top-antitop pair in association with a Higgs boson have been calculated in Refs. [3, 4, 5, 6, 7] and matched to parton showers $[8,9,10]$. Recently electroweak corrections to $t \bar{t} \mathrm{H}$ production have been computed $[11,12,13]$. NLO QCD corrections for the important background processes t $\bar{t} b \bar{b}$ and $t \bar{t} j \mathrm{j}$ production have been worked out in Refs. [14, 15, 16, 17] and Refs. [18, 19, 20], respectively, and matched to parton showers in Refs. [21, 22, 23] and Ref. [24]. In all these calculations the top quarks and the Higgs boson have been treated as stable particles.

In these proceedings we summarise a leading-order study of Higgs-boson production in association with a top-quark pair ( $\mathrm{t} \overline{\mathrm{t}} \mathrm{H})$ including the subsequent semileptonic decay of the top-quark pair and the decay of the Higgs boson into a bottom-antibottom-quark pair, $\mathrm{pp} \rightarrow \mathrm{t} \overline{\mathrm{t}} \mathrm{H} \rightarrow \ell^{+} v_{\ell} \mathrm{jj} \mathrm{j} \overline{\mathrm{b}} \mathrm{b} \overline{\mathrm{b}}$ [25]. We consider this process in three different scenarios, 1) the full process with all Standard Model (SM) Feynman diagrams for the 8-particle final state, 2) t $\bar{t} b \bar{b}$ production, where only diagrams with resonant top-antitop-quark pairs are taken into account, and 3) $\mathrm{t} \overline{\mathrm{t}} \mathrm{H}$ production, where in addition a resonant Higgs boson is required. Comparing the predictions in the three scenarios allows us to examine the size of the irreducible background for Higgs production in association with a top-antitop-quark pair. We have in particular studied different methods of assigning a b-jet pair to the Higgs boson and compared their performance in reconstructing the Higgs signal. Furthermore, we have investigated the size of interference effects between contributions to the matrix elements of different order in the strong and electroweak coupling constants.

We also report on a calculation of the NLO QCD corrections to the hadronic production of a positron, a muon, missing energy, two b jets and a SM Higgs boson, $\mathrm{pp} \rightarrow \mathrm{e}^{+} v_{\mathrm{e}} \mu^{-} \bar{v}_{\mu} \mathrm{b} \overline{\mathrm{b}} \mathrm{H}$ [26], which includes the resonant production of a top-antitop-quark pair in association with a Higgs boson with a subsequent leptonic decay of the top and the antitop quark. Our calculation includes all NLO QCD effects in t⿱ẗH production and top decays and also takes into account all off-shell, non-resonant and interference effects of the top quarks.

\section{Calculational framework}

The full LO process $\mathrm{pp} \rightarrow \ell^{+} v_{\ell} \mathrm{jj} \mathrm{j} b \bar{b} \mathrm{~b} \overline{\mathrm{b}}$ involves partonic channels with up to 78,000 diagrams. All matrix elements are calculated with RECOLA [27] which provides a fast and numerically stable computation. RECOLA uses recursive methods and allows to specify intermediate particles for a given process. The phase-space integration is performed with an in-house multi-channel MonteCarlo program, using phase-space mappings similar to Ref. [28].

We use the complex-mass scheme [29, 30, 31] for the consistent description of all resonances that are not treated in the pole approximation. 
We investigate the cross section and differential distributions for the LHC operating at $13 \mathrm{TeV}$. We employ LHAPDF 6.05 with CT10 parton distributions and neglect contributions from the suppressed bottom-quark parton density and flavour mixing. The electromagnetic coupling $\alpha$ is derived from the Fermi constant in the $G_{\mu}$ scheme. The width of the top quark $\Gamma_{\mathrm{t}}$ is calculated at LO and NLO QCD including effects of off-shell W bosons according to Ref. [32].

We use a standard set of acceptance cuts:

$$
\begin{array}{rrr}
\text { non-b jets: } & p_{\mathrm{T}, \mathrm{j}}>25 \mathrm{GeV}, & \left|y_{\mathrm{j}}\right|<2.5, \\
\mathrm{~b} \text { jets: } & p_{\mathrm{T}, \mathrm{b}}>25 \mathrm{GeV}, & \left|y_{\mathrm{b}}\right|<2.5, \\
\text { charged lepton: } & p_{\mathrm{T}, \ell^{+}}>20 \mathrm{GeV}, & \left|y_{\ell^{+}}\right|<2.5, \\
\text { missing transverse momentum: } & p_{\mathrm{T}, \mathrm{miss}}>20 \mathrm{GeV}, & \\
\text { jet-jet distance: } & \Delta R_{\mathrm{jj}}>0.4, \\
\text { b-jet-b-jet distance: } & \Delta R_{\mathrm{bb}}>0.4, \\
\text { jet-b-jet distance: } & \Delta R_{\mathrm{jb}}>0.4 .
\end{array}
$$

\section{Irreducible background and interference effects in $\mathbf{p p} \rightarrow \ell^{+} v_{\ell} \mathbf{j j b} \overline{\mathbf{b}} \mathbf{b} \overline{\mathbf{b}}$}

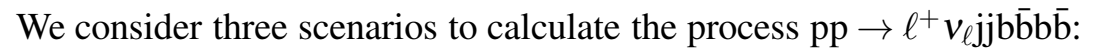

- In the first scenario, the full process, we include all SM contributions to the process $\mathrm{pp} \rightarrow$ $\ell^{+} v_{\ell}$ jjbb̄b $\bar{b}$. Matrix elements involving external gluons receive contributions of $\mathscr{O}\left(\alpha_{\mathrm{s}} \alpha^{3}\right)$, $\mathscr{O}\left(\alpha_{\mathrm{s}}^{2} \alpha^{2}\right)$ and $\mathscr{O}\left(\alpha_{\mathrm{s}}^{3} \alpha\right)$, whereas amplitudes without external gluons receive an additional $\mathscr{O}\left(\alpha^{4}\right)$ term of pure electroweak origin.

- In the second scenario we only take those diagrams into account that contain an intermediate top-antitop-quark pair. The resulting amplitude, labelled $t \bar{t} b \bar{b}$ production in the following, corresponds to the production of a bottom-antibottom pair and an intermediate top-antitop pair followed by its semileptonic decay, i.e. $\mathrm{pp} \rightarrow \mathrm{t} \overline{\mathrm{b}} \mathrm{b} \rightarrow \ell^{+} v_{\ell} \mathrm{jj} \mathrm{j} b \overline{\mathrm{b}} \mathrm{b} \overline{\mathrm{b}}$. Note that we use the pole approximation [33, 34, 35] for the top quarks only, hence we take into account all off-shell effects of the remaining unstable particles. As a consequence of the required top-antitop-quark pair the amplitudes receive no contribution of $\mathscr{O}\left(\alpha_{\mathrm{s}}^{3} \alpha\right)$.

- Finally, we consider the signal process $\mathrm{pp} \rightarrow \mathrm{t} \overline{\mathrm{t}} \mathrm{H} \rightarrow \ell^{+} v_{\ell} \mathrm{jj} \mathrm{j} \overline{\mathrm{b}} \mathrm{b} \overline{\mathrm{b}}$ and label it $\bar{t} H$ production. In addition to the intermediate top-antitop-quark pair we require an intermediate Higgs boson decaying into a bottom-antibottom-quark pair and use the pole approximation for the topquark pair and the Higgs boson. The requirement of the Higgs boson eliminates contributions of $\mathscr{O}\left(\alpha_{\mathrm{s}}^{2} \alpha^{2}\right)$ from the amplitude.

In this analysis we take the bottom quarks massive and use the fixed renormalization and factorization scale according to Ref. [4],

$$
\mu_{\mathrm{fix}}=\mu_{\mathrm{R}}=\mu_{\mathrm{F}}=\frac{1}{2}\left(2 m_{\mathrm{t}}+m_{\mathrm{H}}\right)=236 \mathrm{GeV} .
$$

In Tables 1-3 we present individual contributions to the integrated cross section for the three scenarios. While the first column specifies the partonic initial states $(q=\mathrm{u}, \mathrm{d}, \mathrm{c}, \mathrm{s})$, the following 


\begin{tabular}{llll}
\hline pp & \multicolumn{2}{c}{ Cross section [fb] } \\
& $\mathscr{O}\left(\left(\alpha^{4}\right)^{2}\right)$ & $\mathscr{O}\left(\left(\alpha_{\mathrm{s}} \alpha^{3}\right)^{2}\right)$ & \multicolumn{1}{c}{ Total } \\
\hline$q \bar{q}$ & $0.014887(2)$ & $2.1467(2)$ & $2.1621(2)$ \\
$\mathrm{gg}$ & - & $5.230(1)$ & $5.2298(9)$ \\
\hline$\Sigma$ & $0.014887(2)$ & $7.377(1)$ & $7.3920(9)$ \\
\hline
\end{tabular}

Table 1: Composition of the cross section in $\mathrm{fb}$ for $\mathrm{t} \overline{\mathrm{H}} \mathrm{H}$ production at the $\mathrm{LHC}$ at $13 \mathrm{TeV}$.

\begin{tabular}{lllccc}
\hline pp & \multicolumn{2}{c}{ Cross section [fb] } & & & \\
& $\mathscr{O}\left(\left(\alpha^{4}\right)^{2}\right)$ & $\mathscr{O}\left(\left(\alpha_{\mathrm{s}} \alpha^{3}\right)^{2}\right)$ & $\mathscr{O}\left(\left(\alpha_{\mathrm{s}}^{2} \alpha^{2}\right)^{2}\right)$ & Sum & Total \\
\hline$q \bar{q}$ & $0.018134(6)$ & $2.4932(9)$ & $0.9199(2)$ & $3.4312(9)$ & $3.4366(6)$ \\
$\mathrm{gg}$ & - & $7.818(4)$ & $16.650(9)$ & $24.47(1)$ & $23.010(7)$ \\
\hline$\sum$ & $0.018134(6)$ & $10.311(4)$ & $17.570(9)$ & $27.90(1)$ & $26.446(7)$ \\
\hline
\end{tabular}

Table 2: Composition of the cross section in fb for $t \bar{t} b \bar{b}$ production at the $\mathrm{LHC}$ at $13 \mathrm{TeV}$.

columns contain the contributions resulting from the square of matrix elements of specific orders in the strong and electroweak coupling. The column labelled "Sum" represents the sum of the preceding columns, whereas the last column labelled "Total" provides the integrated cross section including in addition all interference effects between different orders in the couplings.

In Table 1 we show the cross section for $\mathrm{t} \overline{\mathrm{t}} \mathrm{H}$ production and the corresponding contributions resulting from quark-antiquark annihilation and gluon fusion. About $70 \%$ of the events originate from the gluon-fusion process. While the bulk of the contributions results from matrix elements of order $\mathscr{O}\left(\alpha_{\mathrm{s}} \alpha^{3}\right)$, quark-antiquark annihilation receives an additional tiny contribution from pure electroweak interactions. Note that there are no interferences between diagrams of $\mathscr{O}\left(\alpha^{4}\right)$ and $\mathscr{O}\left(\alpha_{\mathrm{s}} \alpha^{3}\right)$ in this scenario.

The composition of the cross section for $t \bar{t} b \bar{b}$ production is shown in Table 2 . We find a significant enhancement of the production rate compared to ttit production, and thus the irreducible background $\sigma_{\mathrm{t} \mathrm{t} b \bar{b}}^{\mathrm{Irred}}=\sigma_{\mathrm{tt} b \overline{\mathrm{b}}}^{\text {Total }}-\sigma_{\mathrm{t} \mathrm{tH}}^{\text {Total }}=19.06 \mathrm{fb}$ exceeds the $\mathrm{t} \overline{\mathrm{t}} \mathrm{H}$ signal by a factor of 2.6. The major contribution to the irreducible background is due to QCD production of $\mathscr{O}\left(\left(\alpha_{\mathrm{s}}^{2} \alpha^{2}\right)^{2}\right)$. The additional contributions of $\mathscr{O}\left(\left(\alpha_{\mathrm{s}} \alpha^{3}\right)^{2}\right)$ in the t⿱t $\mathrm{b} \overline{\mathrm{b}}$ scenario result from Feynman diagrams involving electroweak interactions with $\mathrm{Z}$ bosons, $\mathrm{W}$ bosons and photons, where in particular $\mathrm{t} \overline{\mathrm{Z}} \mathrm{Z}$ production contributes $1.01 \mathrm{fb}$. The difference between the fifth (Sum) and sixth (Total) column in Table 2 is due to interference contributions between matrix elements of different orders in the coupling constants. These cause a reduction of the cross sections by about $5 \%$. The dominant effect is due to interferences of diagrams of $\mathscr{O}\left(\alpha_{\mathrm{s}} \alpha^{3}\right)$ where a W boson is exchanged in the $t$-channel with diagrams of $\mathscr{O}\left(\alpha_{\mathrm{s}}^{2} \alpha^{2}\right)$ that yield the dominant irreducible background. These kinds of interferences are absent in the $q \bar{q}$ channel. On the other hand, we found the interference of the t $\bar{t} H$ signal process with the dominant irreducible background of order $\mathscr{O}\left(\alpha_{\mathrm{s}}^{2} \alpha^{2}\right)$ to be below one per cent. 


\begin{tabular}{lllllll}
\hline pp & \multicolumn{2}{c}{ Cross section [fb] } & & & \\
& $\mathscr{O}\left(\left(\alpha^{4}\right)^{2}\right)$ & $\mathscr{O}\left(\left(\alpha_{\mathrm{s}} \alpha^{3}\right)^{2}\right)$ & $\mathscr{O}\left(\left(\alpha_{\mathrm{s}}^{2} \alpha^{2}\right)^{2}\right)$ & $\mathscr{O}\left(\left(\alpha_{\mathrm{s}}^{3} \alpha\right)^{2}\right)$ & \multicolumn{1}{c}{ Sum } & Total \\
\hline $\mathrm{g} q$ & - & $0.231(4)$ & $0.370(2)$ & $0.365(1)$ & $0.966(4)$ & $0.944(9)$ \\
$\mathrm{g} \bar{q}$ & - & $0.0421(6)$ & $0.0679(3)$ & $0.0608(2)$ & $0.1708(7)$ & $0.167(1)$ \\
$q q^{(\prime)}$ & $0.001471(2)$ & $0.0575(5)$ & $0.1106(2)$ & $0.07871(9)$ & $0.2483(6)$ & $0.2478(8)$ \\
$q \bar{q}$ & $0.01973(3)$ & $2.531(6)$ & $0.957(1)$ & $0.00333(1)$ & $3.511(6)$ & $3.538(4)$ \\
$\mathrm{gg}$ & - & $8.01(2)$ & $17.19(6)$ & $0.00756(2)$ & $25.21(6)$ & $23.71(6)$ \\
\hline$\sum$ & $0.02120(3)$ & $10.87(2)$ & $18.69(6)$ & $0.516(2)$ & $30.10(6)$ & $28.60(6)$ \\
\hline
\end{tabular}

Table 3: Composition of the cross section in fb for the full process at the $\mathrm{LHC}$ at $13 \mathrm{TeV}$. Here $q q^{(\prime)}$ denotes pairs of quarks and/or antiquarks other than $q \bar{q}$.

The results for the full process are listed in Table 3. Here, additional partonic channels ( $\mathrm{g} q, \mathrm{~g} \bar{q}$, $\left.q q^{(\prime)}\right)$ contribute about $5 \%$. The cross section increases by merely $8 \%$ relative to tt̄b $\bar{b}$ production. The contributions of order $\mathscr{O}\left(\left(\alpha_{\mathrm{s}}^{3} \alpha\right)^{2}\right)$ are below $2 \%$ and the interference pattern is similar to the case of $\mathrm{t} t \mathrm{t} \overline{\mathrm{b}}$ production.

In Ref. [25] we investigated the irreducible background and interference effects for various distributions. We found that assigning two $b$ jets to the top- and antitop-quark decay by maximising a combined Breit-Wigner likelihood function and assigning the remaining two $b$ jets to the potential Higgs boson yields a good unbiased determination of the b-jet pair originating from the Higgs-boson decay. While interference effects lead to a constant shift in most of the differential distributions, they cause non-uniform shape changes in a few distributions like the one in the invariant mass of the pair of bottom quarks not resulting from the top quarks and the azimuthal angle between these bottom quarks.

\section{NLO QCD corrections to $\mathbf{p p} \rightarrow \mathbf{e}^{+} v_{\mathrm{e}} \mu^{-} \bar{v}_{\mu} \mathbf{b} \overline{\mathbf{b}} \mathbf{H}$}

We have computed the NLO QCD corrections to the full hadronic process $\mathrm{pp} \rightarrow \mathrm{e}^{+} v_{\mathrm{e}} \mu^{-} \bar{v}_{\mu} \mathrm{b} \overline{\mathrm{b}} \mathrm{H}$ including all resonant, non-resonant, and off-shell effects of the top quarks and all interferences at $13 \mathrm{TeV}$ (for details see Ref. [26]). We include the tree-level amplitudes at $\mathscr{O}\left(\alpha_{\mathrm{s}} \alpha^{5 / 2}\right)$ for gluoninduced and quark-antiquark-induced processes and the corresponding NLO corrections of order $\alpha_{\mathrm{s}}$. The bottom quark is considered massless in this study. The corresponding real corrections receive also contributions of quark-gluon- and antiquark-gluon-initiated processes. We apply the Catani-Seymour subtraction formalism [36, 37] for the regularization and analytical cancellation of IR singularities. We employ RECOLA [27] for the computation of all matrix elements as well as colour- and spin-correlated squared matrix elements needed for the evaluation of subtraction terms.

The matrix elements for the virtual corrections are calculated with RECOLA in dimensional regularisation, which uses the COLLIER [38] library for the numerical evaluation of one-loop scalar $[39,40,41,42]$ and tensor integrals $[43,44,45]$. We sucessfully compared our results for the virtual NLO contribution to the squared amplitude, $2 \operatorname{Re} \mathscr{M}_{0}^{*} \mathscr{M}_{1}$, with MADGrAPH5_AMC@NLO [46]. In 


\begin{tabular}{cccc}
\hline $\mathbf{p p}$ & $\sigma_{\mathbf{L O}}[\mathbf{f b}]$ & $\sigma_{\mathrm{NLO}}[\mathbf{f b}]$ & $K$ \\
\hline $\mathrm{gg}$ & $1.5906(1)_{-23.6 \%}^{+33.7 \%}$ & $2.024(3)_{-16.2 \%}^{+8.4 \%}$ & $1.273(2)$ \\
$q \bar{q}$ & $0.67498(9)_{-18.1 \%}^{+24.1 \%}$ & $0.495(1)_{-39.5 \%}^{+17.2 \%}$ & $0.733(2)$ \\
$\mathrm{g} \bar{q}$ & & $0.136(1)_{-166 \%}^{+295 \%}$ & \\
\hline$\sum$ & $2.2656(1)_{-22.0 \%}^{+30.8 \%}$ & $2.656(3)_{-4.6 \%}^{+0.9 \%}$ & $1.172(1)$ \\
\hline
\end{tabular}

Table 4: Composition of the integrated cross section for $\mathrm{pp} \rightarrow \mathrm{e}^{+} v_{\mathrm{e}} \mu^{-} \bar{v}_{\mu} \mathrm{b} \overline{\mathrm{b}} \mathrm{H}(\mathrm{j})$ at the $13 \mathrm{TeV}$ LHC with the dynamical scale. In column one we list the partonic initial states, where $q=\mathrm{u}, \mathrm{d}, \mathrm{c}, \mathrm{s}$ and $\vec{q}=q, \bar{q}$. The second and third column give the integrated cross sections in fb for LO and NLO, resp., including scale uncertainties. The last column provides the $K$ factor with $K=\sigma_{\mathrm{NLO}} / \sigma_{\mathrm{LO}}$.

addition we checked the Ward identity for the matrix elements of the gluon-initiated process at tree and one-loop level.

We use the anti- $k_{\mathrm{T}}$ algorithm [47] for the jet reconstruction with a jet-resolution parameter $R=0.4$. Only final-state quarks and gluons with rapidity $|y|<5$ are clustered into infrared-safe jets. As default, we use a dynamical scale following Ref. [8],

$$
\mu_{\mathrm{dyn}}=\mu_{\mathrm{R}}=\mu_{\mathrm{F}}=\left(m_{\mathrm{T}, \mathrm{t}} m_{\mathrm{T}, \overline{\mathrm{t}}} m_{\mathrm{T}, \mathrm{H}}\right)^{\frac{1}{3}} \quad \text { with } \quad m_{\mathrm{T}}=\sqrt{m^{2}+p_{\mathrm{T}}^{2}} .
$$

Alternatively, we choose a fixed scale according to Ref. [4] as given in (3.1). Scale uncertainties are determined by computing integrated and differential cross sections at seven scale pairs, $\left(\mu_{\mathrm{R}} / \mu_{0}, \mu_{\mathrm{F}} / \mu_{0}\right)=(0.5,0.5),(0.5,1),(1,0.5),(1,1),(1,2),(2,1),(2,2)$. The central value corresponds to $\left(\mu_{\mathrm{R}} / \mu_{0}, \mu_{\mathrm{F}} / \mu_{0}\right)=(1,1)$, and the error band is constructed from the envelope of these seven calculations.

In Table 4 we present the integrated cross sections for the dynamical scale (4.1). The cross sections for the fixed scale (3.1) are lower by only about $1 \%$, and the $K$ factor for the fixed scale is of 1.176(1). The contribution of the dominating gluon-fusion channel increases from about $70 \%$ at LO to $76 \%$ at NLO. The gluon-(anti)quark induced real-radiation subprocesses contribute about $5 \%$. The inclusion of NLO QCD corrections reduces the scale dependence from $31 \%$ to $5 \%$. In Figure 1 we display the dependence of the integrated LO (blue) and NLO (red) cross sections on the values of the fixed (dashed line) and dynamical scale (solid line) while keeping $\mu_{\mathrm{R}}=\mu_{\mathrm{F}}$. Varying only $\mu_{\mathrm{R}}$ or $\mu_{\mathrm{F}}$ results in smaller variations.

The effects of the finite top-quark width have been determined via a numerical extrapolation to the zero-top-width limit, $\Gamma_{\mathrm{t}} \rightarrow 0$. For fixed scale $\mu_{\mathrm{fix}}$ finite-top-width effects shift the LO and NLO cross section by $-0.07 \pm 0.01 \%$ and $-0.14 \pm 0.22 \%$, respectively, which are within the expected order of $\Gamma_{\mathrm{t}} / m_{\mathrm{t}}$.

We have investigated various differential distributions, two of which are shown in Figure 2 for the dynamical scale choice (4.1). The upper panels show the LO (blue, dashed) and NLO (red, solid) predictions with uncertainty bands from scale variations. The lower panels display the LO (blue) and NLO (red) predictions with scale uncertainties normalized to the LO results at the central scale. Figure 2 a shows the transverse-momentum distribution of the positron. Using the dynamical 


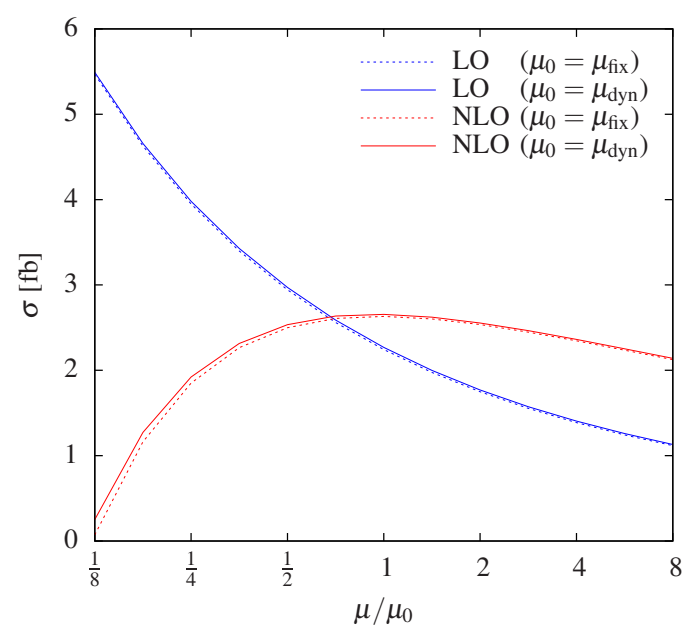

Figure 1: Scale dependence of the LO and NLO integrated cross section at the $13 \mathrm{TeV}$ LHC.
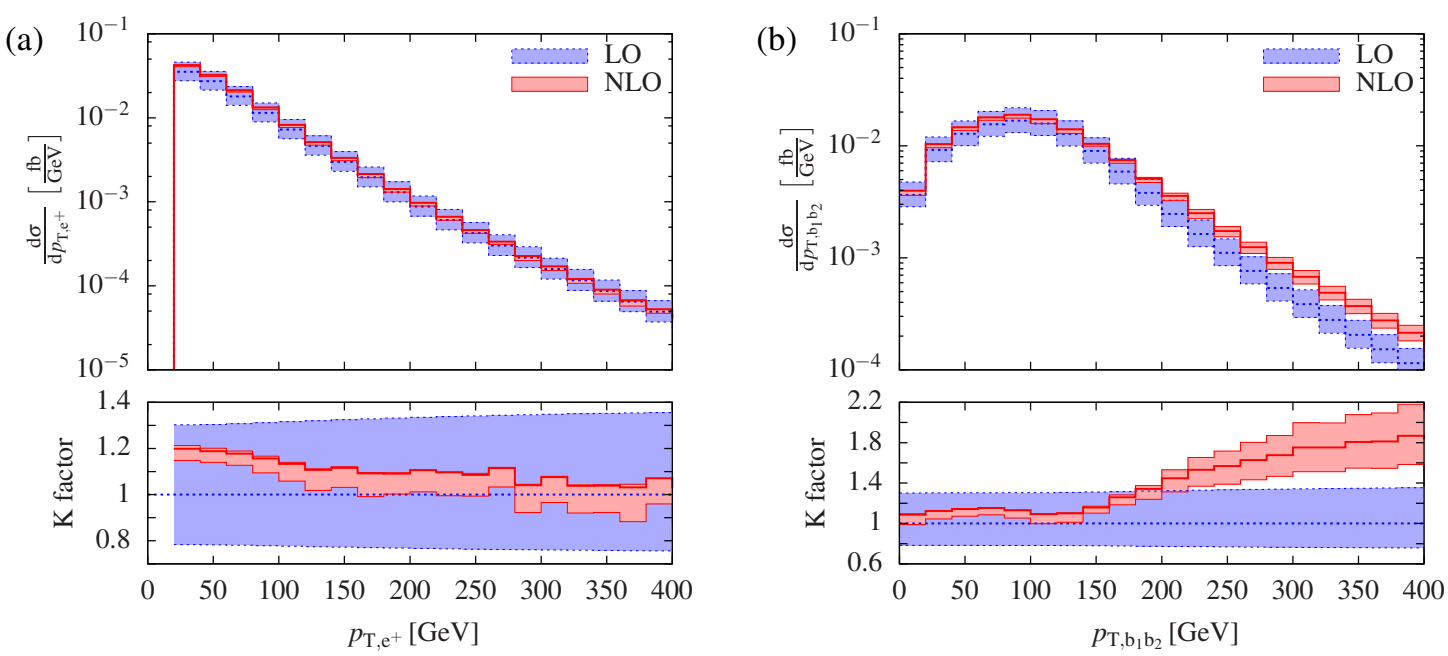

Figure 2: Transverse-momentum distributions at the $13 \mathrm{TeV}$ LHC for the dynamical scale (4.1): (a) for the positron (left), (b) for the b-jet pair (right) and The lower panels show the $K$ factor.

scale, the $K$ factor changes only slightly (within $20 \%$ ) over the displayed range, and the NLO band lies within the LO band. The residual scale variation is at the level of $10 \%$ at NLO. This behaviour is typical for most other distributions (see Ref. [26]). A notable exception is the distribution in the transverse momentum of the b-jet pair (Figure 2b), where we observe an increase of the $K$ factor for high transverse momentum to a value of 1.8 at $p_{\mathrm{T}} \simeq 400 \mathrm{GeV}$. This originates from the fact that this region is suppressed for on-shell top quarks, an effect known already from tt production, where it is even more pronounced [48].

\section{Conclusions}

We have investigated the irreducible background to the production of a Higgs boson decaying 
into bottom quarks in association with a top-antitop-quark pair including its decay. While titH production contributes roughly a fourth to the $\ell^{+} v_{\ell}$ jjb $\bar{b} b \bar{b}$ final state, the major contribution of $92 \%$ is furnished by t $\bar{t} b \bar{b}$ production. Interference effects lower the corresponding cross section by about $5 \%$. We have calculated the next-to-leading-order QCD corrections to off-shell top-antitopquark production in association with a Higgs boson with leptonic decay of the top quarks. Using a dynamical scale, we find $K$ factors mostly in the range 1.0-1.4 and residual scale uncertainties at the level of $10 \%$ for distributions.

\section{Acknowledgments}

This work was supported by the Bundesministerium für Bildung und Forschung (BMBF) under contract no. $05 \mathrm{H} 12 \mathrm{WWE}$.

\section{References}

[1] ATLAS Collaboration, G. Aad et al., Observation of a new particle in the search for the Standard Model Higgs boson with the ATLAS detector at the LHC, Phys.Lett. B716 (2012) 1-29, [arXiv:1207.7214].

[2] CMS Collaboration, S. Chatrchyan et al., Observation of a new boson at a mass of $125 \mathrm{GeV}$ with the CMS experiment at the LHC, Phys.Lett. B716 (2012) 30-61, [arXiv: 1207 . 7235].

[3] W. Beenakker, S. Dittmaier, M. Krämer, B. Plümper, M. Spira, et al., Higgs radiation off top quarks at the Tevatron and the LHC, Phys.Rev.Lett. 87 (2001) 201805, [hep-ph/0107081].

[4] W. Beenakker, S. Dittmaier, M. Krämer, B. Plümper, M. Spira, et al., NLO QCD corrections to $\bar{t} H$ production in hadron collisions, Nucl.Phys. B653 (2003) 151-203, [hep-ph/0211352].

[5] L. Reina and S. Dawson, Next-to-leading order results for $\bar{t} \mathrm{t} H$ production at the Tevatron, Phys.Rev.Lett. 87 (2001) 201804, [hep-ph/ 0107101$].$

[6] S. Dawson, L. Orr, L. Reina, and D. Wackeroth, Associated top quark Higgs boson production at the LHC, Phys.Rev. D67 (2003) 071503, [hep-ph/ 0211438 ].

[7] S. Dawson, C. Jackson, L. Orr, L. Reina, and D. Wackeroth, Associated Higgs production with top quarks at the large hadron collider: NLO QCD corrections, Phys.Rev. D68 (2003) 034022, [hep-ph/0305087].

[8] R. Frederix, S. Frixione, V. Hirschi, F. Maltoni, R. Pittau, et al., Scalar and pseudoscalar Higgs production in association with a top-antitop pair, Phys.Lett. B701 (2011) 427-433, [arXiv:1104.5613].

[9] M. Garzelli, A. Kardos, C. Papadopoulos, and Z. Trócsányi, Standard Model Higgs boson production in association with a top anti-top pair at NLO with parton showering, Europhys.Lett. 96 (2011) 11001, [arXiv:1108.0387].

[10] H. B. Hartanto, B. Jäger, L. Reina, and D. Wackeroth, Higgs boson production in association with top quarks in the POWHEG BOX, Phys. Rev. D91 (2015), no. 9 094003, [arXiv: 1501.04498 ].

[11] S. Frixione, V. Hirschi, D. Pagani, H. S. Shao, and M. Zaro, Weak corrections to Higgs hadroproduction in association with a top-quark pair, JHEP 1409 (2014) 065, [arXiv:1407.0823]. 
[12] Z. Yu, M. Wen-Gan, Z. Ren-You, C. Chong, and G. Lei, QCD NLO and EW NLO corrections to $\bar{t} \bar{t} H$ production with top quark decays at hadron collider, Phys.Lett. B738 (2014) 1-5, [arXiv:1407.1110].

[13] S. Frixione, V. Hirschi, D. Pagani, H. S. Shao, and M. Zaro, Electroweak and QCD corrections to top-pair hadroproduction in association with heavy bosons, arXiv:1504.03446.

[14] A. Bredenstein, A. Denner, S. Dittmaier, and S. Pozzorini, NLO QCD corrections to ț̄b $\bar{b}$ production at the LHC: 1. Quark-antiquark annihilation, JHEP 0808 (2008) 108, [arXiv: 0807 . 12 48].

[15] A. Bredenstein, A. Denner, S. Dittmaier, and S. Pozzorini, NLO QCD corrections to $p p \rightarrow \bar{t} b \bar{b}+X a t$ the LHC, Phys.Rev.Lett. 103 (2009) 012002, [arXiv: 0905 . 0110].

[16] G. Bevilacqua, M. Czakon, C. Papadopoulos, R. Pittau, and M. Worek, Assault on the NLO Wishlist: $p p \rightarrow \bar{t} b \bar{b}$, JHEP 0909 (2009) 109, [arXiv : 0907 . 4723].

[17] A. Bredenstein, A. Denner, S. Dittmaier, and S. Pozzorini, NLO QCD Corrections to Top Anti-Top Bottom Anti-Bottom Production at the LHC: 2. full hadronic results, JHEP 1003 (2010) 021, [arXiv: 1001.4006$]$.

[18] G. Bevilacqua, M. Czakon, C. G. Papadopoulos, and M. Worek, Dominant QCD Backgrounds in Higgs Boson Analyses at the LHC: A Study of pp $\rightarrow t \bar{t}+2$ jets at Next-To-Leading Order, Phys. Rev. Lett. 104 (2010) 162002, [arXiv: 1002 . 4009 ].

[19] G. Bevilacqua, M. Czakon, C. G. Papadopoulos, and M. Worek, Hadronic top-quark pair production in association with two jets at Next-to-Leading Order QCD, Phys. Rev. D84 (2011) 114017, [arXiv:1108.2851].

[20] G. Bevilacqua and M. Worek, On the ratio of $t \bar{t} b \bar{b}$ and $t \bar{t} j j$ cross sections at the CERN Large Hadron Collider, JHEP 07 (2014) 135, [arXiv: 1403.2046$].$

[21] A. Kardos and Z. Trócsányi, Hadroproduction of t anti-t pair with a b anti-b pair using PowHel, J.Phys. G41 (2014) 075005, [arXiv: 1303 . 6291].

[22] F. Cascioli, P. Maierhöfer, N. Moretti, S. Pozzorini, and F. Siegert, NLO matching for $\bar{t} \bar{b} \bar{b}$ production with massive b-quarks, arXiv:1309.5912.

[23] M. V. Garzelli, A. Kardos, and Z. Trócsányi, Hadroproduction of ṫ̄b $\bar{b}$ final states at LHC: predictions at NLO accuracy matched with Parton Shower, JHEP 03 (2015) 083, [arXiv: 1408 . 0266 ].

[24] S. Höche, F. Krauss, P. Maierhöfer, S. Pozzorini, M. Schönherr, and F. Siegert, Next-to-leading order QCD predictions for top-quark pair production with up to two jets merged with a parton shower, Phys. Lett. B748 (2015) 74-78, [arXiv: 1402 . 6293].

[25] A. Denner, R. Feger, and A. Scharf, Irreducible background and interference effects for Higgs-boson production in association with a top-quark pair, JHEP 1504 (2015) 008, [arXiv: 1412 . 5290].

[26] A. Denner and R. Feger, NLO QCD corrections to off-shell top-antitop production with leptonic decays in association with a Higgs boson at the LHC, arXiv: 1506.07448.

[27] S. Actis, A. Denner, L. Hofer, A. Scharf, and S. Uccirati, Recursive generation of one-loop amplitudes in the Standard Model, JHEP 1304 (2013) 037, [arXiv: 1211.6316$].$

[28] S. Dittmaier and M. Roth, LUSIFER: A LUcid approach to six FERmion production, Nucl.Phys. B642 (2002) 307-343, [hep-ph/0206070].

[29] A. Denner, S. Dittmaier, M. Roth, and D. Wackeroth, Predictions for all processes $e^{+} e^{-} \rightarrow 4$ fermions $+\gamma$,Nucl.Phys. B560 (1999) 33-65, [hep-ph / 9904472 ]. 
[30] A. Denner, S. Dittmaier, M. Roth, and L. Wieders, Electroweak corrections to charged-current $e^{+} e^{-} \rightarrow 4$ fermion processes: Technical details and further results, Nucl.Phys. B724 (2005) 247-294, [hep-ph / 05050 42]. Erratum-ibid. B854 (2012) 504-507.

[31] A. Denner and S. Dittmaier, The Complex-mass scheme for perturbative calculations with unstable particles, Nucl.Phys.Proc.Suppl. 160 (2006) 22-26, [hep-ph / 0605312$].$

[32] M. Jezabek and J. H. Kühn, QCD corrections to semileptonic decays of heavy quarks, Nucl.Phys. B314 (1989) 1.

[33] R. Kleiss and W. J. Stirling, Top quark production at hadron colliders: some useful formulae, Z.Phys. C40 (1988) 419-423.

[34] R. G. Stuart, Gauge invariance, analyticity and physical observables at the Z0 resonance, Phys.Lett. B262 (1991) 113-119.

[35] A. Aeppli, G. J. van Oldenborgh, and D. Wyler, Unstable particles in one loop calculations, Nucl.Phys. B428 (1994) 126-146, [hep-ph/9312212].

[36] S. Catani and M. Seymour, A General algorithm for calculating jet cross-sections in NLO QCD, Nucl.Phys. B485 (1997) 291-419, [hep-ph/ 9605323$].$

[37] S. Catani, S. Dittmaier, M. H. Seymour, and Z. Trócsányi, The Dipole formalism for next-to-leading order QCD calculations with massive partons, Nucl.Phys. B627 (2002) 189-265, [hep-ph/0201036].

[38] A. Denner, S. Dittmaier, and L. Hofer, COLLIER - A fortran-library for one-loop integrals, PoS LL2014 (2014) 071, [arXiv: 1407 . 0087].

[39] G. 't Hooft and M. J. G. Veltman, Scalar One Loop Integrals, Nucl. Phys. B153 (1979) 365-401.

[40] W. Beenakker and A. Denner, Infrared divergent scalar box integrals with applications in the electroweak standard model, Nucl. Phys. B338 (1990) 349-370.

[41] S. Dittmaier, Separation of soft and collinear singularities from one-loop N-point integrals, Nucl. Phys. B675 (2003) 447-466, [hep-ph / 0308246 ].

[42] A. Denner and S. Dittmaier, Scalar one-loop 4-point integrals, Nucl. Phys. B844 (2011) 199-242, [arXiv:1005.2076].

[43] G. Passarino and M. J. G. Veltman, One Loop Corrections for $e^{+} e^{-}$Annihilation Into $\mu^{+} \mu^{-}$in the Weinberg Model, Nucl. Phys. B160 (1979) 151.

[44] A. Denner and S. Dittmaier, Reduction of one-loop tensor 5-point integrals, Nucl. Phys. B658 (2003) 175-202.

[45] A. Denner and S. Dittmaier, Reduction schemes for one-loop tensor integrals, Nucl. Phys. B734 (2006) 62-115.

[46] J. Alwall, R. Frederix, S. Frixione, V. Hirschi, F. Maltoni, et al., The automated computation of tree-level and next-to-leading order differential cross sections, and their matching to parton shower simulations, JHEP 1407 (2014) 079, [arXiv: 1405 .0301].

[47] M. Cacciari, G. P. Salam, and G. Soyez, The Anti-k(t) jet clustering algorithm, JHEP 0804 (2008) 063, [arXiv:0802.1189].

[48] A. Denner, S. Dittmaier, S. Kallweit, and S. Pozzorini, NLO QCD corrections to off-shell top-antitop production with leptonic decays at hadron colliders, JHEP 1210 (2012) 110, [arXiv: 1207.5018 ]. 